\title{
Histochemical characterization of human osteochondral tissue: comparison between healthy cartilage, arthrotic tissues, and cartilage defect treated with $\mathrm{MACl}$ technique
}

\author{
F. Tessarolo'*, R. Tatti', M. Molinari², P. Dorigotti', R. Antolini', G. Nollo' \\ I Interdepartmental Centre on Biomedical Technologies, University of Trento, via delle Regole 101, \\ 38123 Mattarello, Trento, Italy \\ ${ }^{2}$ Dept. of Orthopaedics and Traumatology, APSS, viale De Gasperi 79, 38/23, Trento, Italy \\ * tessaro@science.unitn.it
}

KEYWORDS: $\mathrm{MACl}$, cartilage, knee, chondrocytes, histochemistry

\begin{abstract}
Matrix-induced autologous chondrocytes implantation (MACI) is a promising technique for the treatment of articular cartilage lesions, but long time outcome have to be established. We developed and optimized specific techniques of histochemical staining to characterize healthy and pathologic osteochondral tissue. Seven different staining protocols were applied to assess tissue architecture, cells morphology, proteoglycan content, and collagen fibers distribution. Potentialities of histochemical staining and histomorphology of biopsies from second look arthroscopy will be presented.
\end{abstract}

\section{Introduction}

Surgical therapies for the treatment of articular cartilage lesions can be grouped into reparative and restorative techniques. Clinical results showed reparative techniques lead to the formation of fibrocartilagineous tissue. Differently, restorative procedures can repair the osteochondral defects with a hyaline-like cartilage [1]. In last years, autologous chondrocytes implantation after in vitro expansion has been further improved by $\mathrm{MACl}$ (Matrix induced autologous implantation chondrocytes) technique [2], using three-dimensional scaffolds made of bioresorbable biomaterials. To assess the efficacy of long-term treatment, the histological evaluation of osteochondral tissue plays a key role in the distinction between hyaline cartilage and fibrocartilage. The study aimed at evaluating and optimizing histochemical staining protocols to compare healthy and arthrotic osteochondral tissue to regenerated tissue after $\mathrm{MACl}$.

\section{Material and Methods}

Three sample types of human osteochondral tissue were considered: healthy (lateral femoral condyle, female, 78 years), arthrotic (tibial plate, female, 84 years), and regenerated with $\mathrm{MACl}$ technique (lateral femoral condyle, male, 51 years, biopsy obtained during second-look arthroscopic surgery in symptomatic patient, 1 year after $\mathrm{MACl})$. Tissues were fixed in $10 \%$ buffered formalin for $24 \mathrm{~h}$, decalcified in EDTA for 24h, embedded in paraffin, and cut transversally in $3-5 \mu \mathrm{m}$ slides. Sections were subjected to one of the following protocols: Hematoxylin-Eosin $Y$ (H\&E), Safranin-O (SO), Alcian Blue pH2.5 (AB), Toluidine Blue (TB), Astra Blue pH2.5 (AsB), Azan (Az) and Picrosirius Red (PR). Stained sections were dehydrated, mounted with acrylic medium and observed at magnifications ranging from 40 to 500 times in white and polarized light. Tissue sections were evaluated for surface regularity, proteoglycan content, spatial distribution of chondrocytes and collagen fibers orientation.

\section{Results}

H\&E successfully characterized morphology, and spatial organization of the chondrocytes. SO,AB,AsB,TB stained proteoglycans. According to its specificity [3], SO was more effective than Toluidine Blue in revealing proteoglycan depletion in pathologic tissue. $A B$ and $A s B$ staining can be affected by $\mathrm{pH}$ and temperature. Collagen fibers were stained by both $A z$ and $P R$, but the latter protocol was less complex and samples can be viewed also in polarized light.

\section{Discussion}

The comparison of healthy and arthrotic tissue showed cartilage wear and surface fibrillation. (fig.1a, 1b).

Regenerated tissue showed to be fibrocartilagineous in the chondral side (depletion of the cationic staining, misalignment of the chondrocytes distribution and loss of normal orientation in collagen fibers) and hyaline-like in the bone side (Fig. 1c). Moreover a deep tear, compromising 
Histochemical characterization of human osteochondral tissue: comparison between healthy cartilage, arthrotic tissues,

tissue integrity, was found between the two layers, showing a non complete regeneration of the lesion.

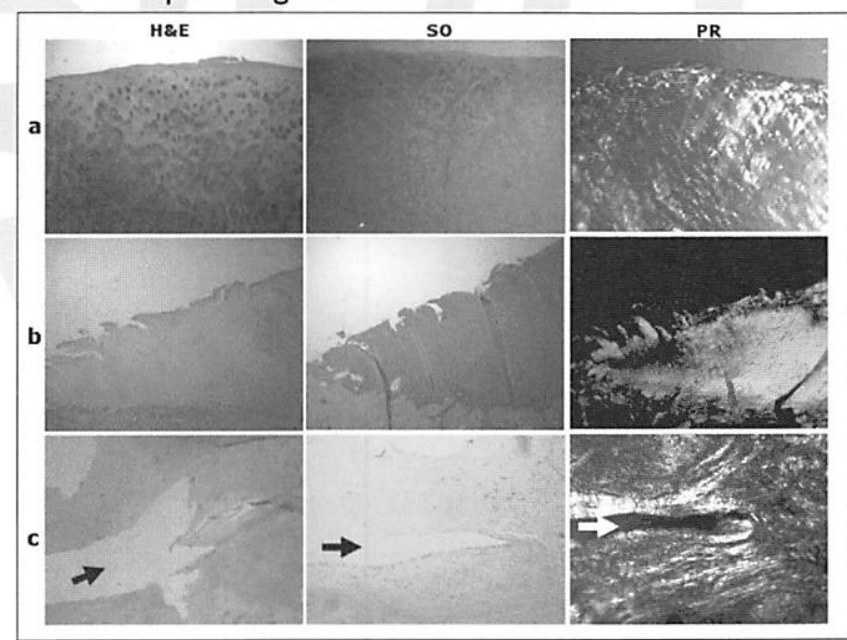

Figure I. (a) healthy, (b) arthrotic, and (c) regenerated osteochondral tissue. PR was obtained in polarized light. Arrows indicate tissue tear. Original magnification: 40x ( $a$ and b), 200x (c).

\section{References}

[1] Shah M.R., Kaplan K.M., Meislin R.J., Bosco J.A. 2007.Articular cartilage restoration of the knee. Bulletin of the NYU Hospital for joint diseases., 65: 51-60.

[2] Marlovits S., Striessnig G., Kutscha-Lissberg F., Resinger C., Aldrian S.M.,Vécsei V., Trattnig S. 2005. Early postoperative adherence of matrix - induced autologous chondrocyte implantation for the treatment of fullthickness cartilage defects of the femoral condyle. Knee Surg Sports Traumatol Arthrosc., 13: 451-457.

[3] Hyllested J.L.,Veje K., Ostergaard K. 2002. Histochemical studies of the extracellular matrix of human articular cartilage-a review. Ostheoarthritis and Cartilage, 10: 333-343. 